\title{
Adaptation of a Smoking Cessation and Prevention Website for Urban American Indian/Alaska Native Youth
}

\author{
Maile Taualii • Nigel Bush • Deborah J. Bowen • \\ Ralph Forquera
}

Published online: 16 January 2010

(C) The Author(s) 2009

\begin{abstract}
Tobacco use among American Indian youth is a disproportionately significant problem. We adapted and modified an existing web-based and youth-focused tobacco control program to make it appropriate for young urban American Indian/Alaska Natives (AI/ANs). The results of the focus group indicate that $\mathrm{AI} / \mathrm{AN}$ youth were very receptive to the use of a web-based Zine-style intervention tool. They wanted the look and feel of the website to be more oriented toward their cultural images. Future research should examine if successful programs for reducing non-ceremonial tobacco use among urban AI/AN youth can keep young irregular smokers from becoming adult smokers.
\end{abstract}

This study was funded as a supplement to the Spirit of Eagles Special Populations Network under subcontract CA86098-05N (N. Bush/J.

Kaur). The project was a collaborative venture between the Urban Indian Health Institute, a division of the Seattle Indian Health Board, the Fred Hutchinson Cancer Research Center, and Native People for Cancer Control. The authors also would like to acknowledge the assistance of the NCI's Cancer Information Service Northwest Region in initiating this research collaboration and providing expertise throughout.

M. Taualii $\cdot$ R. Forquera

Seattle Indian Health Board and the Urban Indian Health Institute, Seattle, USA

N. Bush • D. J. Bowen

Fred Hutchinson Cancer Research Center,

Seattle, USA

N. Bush

National Cancer Institute's Cancer Information Service,

Bethesda, Maryland, USA

\section{J. Bowen $(\triangle)$}

Boston University, Department of Community Health Sciences, 801 Massachusetts Avenue, Crosstown Center, 4th floor,

Boston, MA 02124, USA

e-mail: dbowen@bu.edu
Keywords Native American Youth · Tobacco Use · eHealth Intervention

\section{Introduction}

Tobacco use is responsible for more premature morbidity and mortality than any other behavioral risk factor $[1,2]$ and rates of smoking are particularly high (40\%) among American Indians and Alaska Natives (AI/AN) [1-7]. Of concern, is that while tobacco use has declined among the general US population, smoking prevalence among AI/AN people remains high $[8,9]$. In contrast to whites, smoking rates do not decline with increasing education and income among AI/ANs [10].

Among adolescent AI/AN, rates of smoking are higher than for Caucasian youth $[11,12]$. Data from a Bureau of Indian Affairs high school survey and the 2001 Youth Risk Behavior Study show more AI/AN youth had ever smoked ( $88 \%$ vs. $64 \%)$ and a study with Bureau of Indian Affairs middle-school students revealed $73 \%$ had ever tried cigarettes [13].

The Indian Health Care Improvement Act [14] defines "Urban Indians" as AI/ANs residing in urban centers who are likely members or descendants of many different tribes and who may or may not maintain cultural, religious, or historical ties. Evidence shows that tobacco use starts in youth and that urban $\mathrm{AI} / \mathrm{AN}$ youth is especially vulnerable. While access to tobacco on Indian reservations may be easier for Indian youth because state regulations regarding the age at which a minor can purchase cigarettes do not apply, access for urban Indians is no different than for others. Although two-thirds of the people who describe themselves solely or partially as AI/AN live in metropolitan areas, there has been little attention given to the health of Indian people living in 
American cities [14-16]. Only $1 \%$ of the federal Indian Health Service budget is allocated for non-reservation mostly "urban" Indians although two-thirds of Indian people are now living in American cities [17]. It is imperative that efforts to curb the use of tobacco devise strategies that are accessible to AI/AN youth and that the messages are communicated in a way that is culturally respectful yet fitting to the social setting where these youth live.

The Worldwide Web has been proposed as a delivery vehicle for smoking cessation support by providing information, discussion groups, cognitive behavioral treatment, and self-help materials [18-28]. Moreover, online tobacco control efforts have emerged [29-36], but none have been designed for urban AI/AN youth. The purpose of this formative study, therefore, was to collect data on AI/AN youth's ideas about tobacco and to use those ideas to modify an existing webbased youth smoking prevention and cessation resource, SmokingZine [27, 37-39], for urban AI/AN youth.

\section{Methods}

This two-phase pilot study was a collaborative project between the Urban Indian Health Institute (a division of the Seattle Indian Health Board), the Fred Hutchinson Cancer Research Center, and Native People for Cancer Control, an NCI-funded group of investigators and community members focused on improving the cancer-related health $\mathrm{AI} / \mathrm{AN}$ people in the Pacific Northwest. Phase 1 involved reviewing and adapting an existing web-based program for urban AI/AN youth. Using a focus group format, we modified text, graphics, and functionality based on input from youth from a single urban AI/AN community. In phase 2, we compared the adapted and original websites in a second sample of $\mathrm{AI} / \mathrm{AN}$ youth.

Test Website Since 1995, the TeenNet project at the University of Toronto has combined website development, community mobilization, and action research involving young people from diverse backgrounds in designing, developing, and disseminating programs [40]. In 2002, TeenNet began a randomized, controlled trial of SmokingZine, our test website [27, 37-39]. This large trial of an ehealth intervention targeting behavior change involved over 1,400 adolescents in Toronto area high schools in a smoking cessation intervention. We used the SmokingZine as the starting place for our adaptation.

Participant Recruitment Our sample consisted of youth between the ages of 12 and 18 years identified by the Urban Indian Health Institute as urban AI/ANs. We selected this age range to encompass a group young enough to derive persisting benefits from the intervention, yet mature enough both to respond meaningfully and to encourage riskreducing behavior among peers and family members.

We recruited participants using flyers posted at the Seattle Indian Health Board, other organizations serving $\mathrm{AI} / \mathrm{AN}$ youth, and local powwows. Interested youth called project staff who screened callers for eligibility and described the study. We strove to recruit both sexes and equally represent age categories. Consent or assent forms, completed by parents for youth under 18 years of age, were obtained from all participants. Participants received a $\$ 20$ gift certificate.

Phase 1 Procedures We conducted a 2-h session during which youth completed a short baseline survey on demographics, technology, and tobacco use, participated in a 45-min focus group, and reviewed the original SmokingZine website. After the focus group, participants viewed and discussed a PowerPoint presentation of the original SmokingZine website. Transcripts were entered into NUD*IST, a software package that compiles and analyzes non-numerical, unstructured, qualitative, and text-based data using techniques of indexing, searching, and theorizing about observed patterns. After the transcript codes were entered into NUD*IST, all data for a given code was retrieved in a single report. From these reports, emergent themes were explored in depth and the range of views expressed within a theme, as well as the relationship between themes, was considered.

Phase 2 Procedures In phase 2, we tested usability of the modified website and compared it with the usability of the original website. We used a formalized and well-established usability test for evaluating websites at various stages of development with diverse audiences available at usability. gov and used in several web-based research projects at the Fred Hutchinson Cancer Research Center. Usability testing involved measuring and analyzing the quality of the $\mathrm{AI} / \mathrm{AN}$ youths' experience when interacting with the original and adapted versions of the website, focusing on refining cultural relevance, functionality, organization of content, and ease of navigation and use of website tools such as the SmokingZine quiz where participants provided answers to questions that were combined into a tailored page about their smoking behaviors.

\section{Results}

Phase 1

We recruited five male and seven females for the focus groups (six members each). Five were 13-14, two were 15-16, and 
five were 17-18 years of age. Participants were distributed as evenly as possible by age and gender across the two focus groups. As mentioned previously, urban Indian communities represent a wide array of experiences and tribal affiliations. Tribal affiliations cited by the 12 focus group members included: Ponca, Assinaboin, Cree, Shoshone, Yakama, Shoshone, Gros Vente, Sioux, Caddo, Blackfeet, Makah, Lakota Sioux, and Aztec. Six participants were past smokers. A number of themes, described in detail below, emerged from the phase 1 focus group discussions concerning both tobacco issues and revision of the SmokingZine website for $\mathrm{AI} / \mathrm{AN}$ youth. Table 1 summarizes those themes.

Computer/Internet Skills and Use Most youth rated their computer skills at the beginner level, their internet skills as intermediate, and most used computers, internet, or email at least once a day, most often at home or school. The majority used the internet for email, chat rooms, to play games or for school or personal research. Participants identified two main uses for computers: reports/papers for school and for jobs that involved computer use (for example, office work). Online shopping was the most cited reason for using the internet followed by information gathering (for school or other), expressing oneself (ediaries, blogs, and website development), and for email. Health information or information on smoking or tobacco was not commonly cited as a reason for accessing the internet. All youth felt that information from the internet was useful, though its' trustworthiness was questioned.
Tobacco Use Youth talked about the use of ceremonial tobacco for blessing drums, as something to offer up to the spirits, and in sweats. Several themes emerged when participants were asked about non-ceremonial tobacco use. Stress relief was commonly cited by both focus group participants. Many youth identified that as the reason for their parent's or family member's tobacco use. Additionally, youth said people smoke to look or be cool.

“...I've been smoking for almost ten years just to be cool”. M5

Tobacco use was also reported by a couple of youth to be an appetite suppressant, which was listed as a reason adolescents smoked.

Two themes emerged when participants were asked reasons on why people do not use tobacco. The most cited reason was to avert sickness and the other was because "it's stupid". Participants identified cigarettes and chewing tobacco as the only two ways to use tobacco. Participants named seven brand names of cigarettes more than once, with Desert Sun being the most cited followed by Marlboro, Camels, Menthols, Native Spirits, Carnivals, and Rogers.

Tobacco Use by Other People In the participant's communities, most people smoked around school, in the car, in the house, on the streets, or at bars. Places participants acknowledged as being smoke-free include hospitals, in some houses, inside public buildings, and stores. Most

Table 1 Themes identified from phase 1 focus groups

\begin{tabular}{ll}
\hline Themes & Examples \\
\hline Smoking advertisement & Where they were seen-billboards, in magazines, or through promotional methods, like coupons \\
Reasons for non-ceremonial tobacco use & Who was advertising - traditional tobacco use positive v commercial use negative \\
& Stress relief \\
& To look or be cool \\
& Appetite suppressant \\
& Peer pressure \\
& Self-destructive behavior \\
& To avert sickness \\
& Because "it's stupid" \\
Reasons not to start using tobacco & To avert sickness or death \\
Reasons to quit using tobacco & Fear of getting into trouble (by parents) \\
& Cost (too expensive) \\
& Change color scheme and fonts \\
Website redesign tailored to AI/AN youth & Use more pictures of real things and people instead of animation \\
& Change all graphics to be specific to Native culture \\
Add/change content to reflect Native customs & Distinguish between tobacco use for ceremonial and non-ceremonial use \\
Recognize ceremonial uses of tobacco as positive and important to the heritage of Native people
\end{tabular}


participants encountered second-hand smoke in outside public areas (like the park), at home, or in the car. Reasons most cited for American Indian/Alaska Natives starting to smoke include stress, peer pressure, and self-destructive behavior.

Tobacco Use Prevention and Initiation In general, participants felt that anti-smoking campaigns do not work. "... people using that crap don't care so how they gonna quit?" M3

Two themes emerged when participants were asked whether they had seen or heard smoking advertisements. The first was places where they were seen and the other was who was advertising. The most commonly reported places that participants had seen smoking advertisements were on billboards, in magazines, or through promotional methods like coupons being sent to their house.

"They give you, like the promotional things, like where they give you camel dollars. They used to, I don't know if they do it now, but if you save up enough you can get like a magazine." M8

"...or if you save one of those things from like Marlboro, you can send them or something...remember?" M6

Traditional tobacco use was the only positive use of tobacco referenced by both focus groups. Participants stated that the negative aspects of tobacco use were all related to negative health outcomes associated with smoking.

Quitting Smoking The most commonly cited reasons for people to stop smoking were to avert sickness or death, fear of getting into trouble (by parents), and because of the cost (too expensive). The two reasons cited by participants for those who do not quit smoking were because they were addicted or because a person had no reason to quit. Methods people used to stop smoking reported by participants included using patches, gum, non-tobacco cigarettes, utilizing outside sources for help (doctors and therapists), and they stop altogether. Resources smokers turn to quit smoking included the tobacco helpline, doctors, family, and the internet.

Entertainment Many participants identified sports as something they did for fun, as well as writing (journals), hanging out with friends, and going to school. Two themes emerged when youth were asked about things they liked to buy. The first was about things that they actually bought, such as clothes, food, and shoes. The second theme was things they would buy if they could and included things like cars, stereos, and concert tickets.
If I had enough money, I would buy a stereo or something...(M5)

Almost every participant identified powwows as an Indian Community event that they attended. Several participants were also involved in a Native acting group and dancing. A couple participants identified gambling as an Indian Community event that they or someone they know attended.

Phase 2

Thirteen youth, 12-18 years of age, participated in the usability testing of both the original SmokingZine website and the modified AI/AN website (Table 2).

Ease of Use/Ease of Understanding For most website components or sections, participants rated both versions either somewhat or very easy and in all cases moderately in favor of the AI/AN version. The same was true of ratings of how easy each component was to understand.

"It was cool and creative"-17-year-old female

"Direct comparison things you can have your whole life, or you could just smoke it up."-17-year-old male

"You choose your decisions and can see it visually. It makes you admit, helps make your own decisions with out others' criticism. Allows you to commit to yourself."-18-year-old female

"You have the choices and if you don't accept the choices it is hard, but you can do it - it doesn't hurt to try."-15-year-old female

Improvements We accrued very specific suggestions for small-scale improvements to each page and section of the $\mathrm{AI} / \mathrm{AN}$ site which will be implemented in the next iteration. A few representative examples of many show the level of detail provided by the participants:

"Move to feather 5 keep numbers, text, at top and put the list below, add to the list, "no good things/ concerns."-13-year-old female

"Add a title that says "what you answered" or "what you said" - might also mention that the decisions you make will affect not only you but others around you (second hand smoke). Consider how others will be affected."-18 year-old female

"Add option to email to a friend instead of self. I like the 'hot talk' option, and the links."-18-year-old female 
"You should show pictures of what you could be doing instead of smoking." -15 -year-old female

Cessation The consensus from the recorded narrative seemed to be that the site might help with tobacco prevention or cessation. For example: "The site would help kids that need help with
smoking."-17-year-old male

"The site will make the person look at themselves both smokers and non-smokers."-18-year-old female

"It makes you want to do it" [quit smoking]"-13-year-old male

\section{Discussion}

The purpose of this study was to collect data on urban AI/ AN youth's ideas about tobacco and to use those ideas to modify an existing web-based youth smoking prevention and cessation resource, SmokingZine [27, 37-39], for urban AI/AN youth. We collected the tobacco-related ideas via a focus group of urban AI/AN youth, and used those ideas to modify the SmokingZine, an existing and evaluated website. We then evaluated the adapted website with another focus group of youth. Specifically, we found that many of the same ideas about tobacco that urban youth from dominant culture populations hold (e.g., ideas about advertising, tobacco-related images, etc) were also held by urban AI/AN youth. These ideas were kept in the adapted version of the website. However, the look and feel of the website was changed dramatically based on feedback from the first focus group, to be more "Native"- "Add music and graphics - native music, not white man music."-17-yearold female"-and to work well with multiple AI/AN cultures and nations represented in the focus group. This concept of "Nativeness" as an identity pervaded the focus group discussions, epitomized by one group member who objected to the use of the word "Indian" to describe himself because "American Indian ...reminds me of, like, Indians in Russia and stuff over that way and Native American reminds me of just native people." By Incorporating the cultural distinctions defined by the first focus group, the adaptation process produced a new website that was acceptable and valued by youth from the second focus group.

We are aware of one tobacco program targeting AI youth. In this school-based project, which used a culturally derived curriculum, the goals of tobacco use prevention were achieved in a large cohort of AI youth [41]. Although many factors are important in designing tobacco interven- tions, effective instructional approaches favor participatory or interactive teaching strategies over traditional didactic instruction and provide concentrated instruction [42-45]. A recent meta-analyses of 207 school-based drug prevention programs conducted between 1978 and 1998 highlights the importance of an interactive component [46]. Interactive programs produced greater effect sizes than the noninteractive programs across the board for all drug abuse programs.

The "one size fits all" approach to tobacco prevention programs might not be as effective as programs that are more culturally matched to their audiences [47]. In a pertinent study, investigators compared the effectiveness of a generic skills training program, a culturally focused intervention approach, and an information-only control group on various types of drug use [44]. At 2 years, the culturally tailored intervention had greatest impact on alcohol consumption. Others have evaluated the effects of a school-based, multicultural curriculum among sixth grade students in 16 ethnically diverse schools in Southern California and found that the multicultural program had lower smoking initiation rates compared to a standard curriculum only for Hispanic boys [48].

The importance of culture has also been recognized as a factor in effective learning. For AI/AN youth, a positive learning atmosphere is created through the viewing of cultural differences as strengths [49], and a culturally appropriate curriculum may empower AI/AN students and enhance their education [50]. Lastly, several studies have demonstrated that AI students more often processed information visually more than verbally [51-53] and others have observed they responded better to visual than verbal instruction [49]. Of note, the Indian Nations at Risk Task Force has pointed out the negative effects of "passive transmission" teaching methods for Native students [54]. Taken together, these findings suggest our visual, interactive AI/AN SmokingZine website may be more effective with AI/AN youth than conventional approaches.

This study has several notable limitations. First, it was conducted at only one urban site with youth of a restricted age range. Participants in the study may be representatives of a specific tribe; however, given the unique culture and history of Urban Indian communities readers should not generalize findings from this study to the specific tribe any of the participants are associated with. Instead, the reader should interpret and apply findings to the 2.8 million or $67 \%$ of the Indian population no longer on reservation, who may or may not have one specific tribal affiliation. Second, although not uniformly true, access to computers is not ubiquitous in settings with $\mathrm{AI} / \mathrm{AN}$ settings, and so the choice of intervention delivery system has drawbacks. The youth in this study appeared satisfied with the website too, however, suggesting that technologies such as the one 


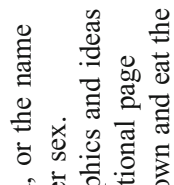

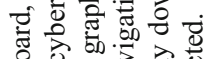

员

둥

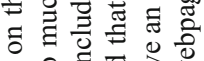

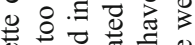

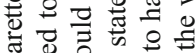

.

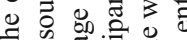

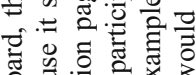

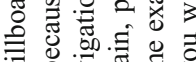

ว

Ð

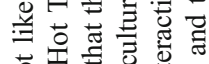

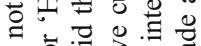

풍 记

늘

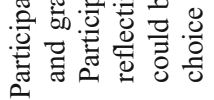
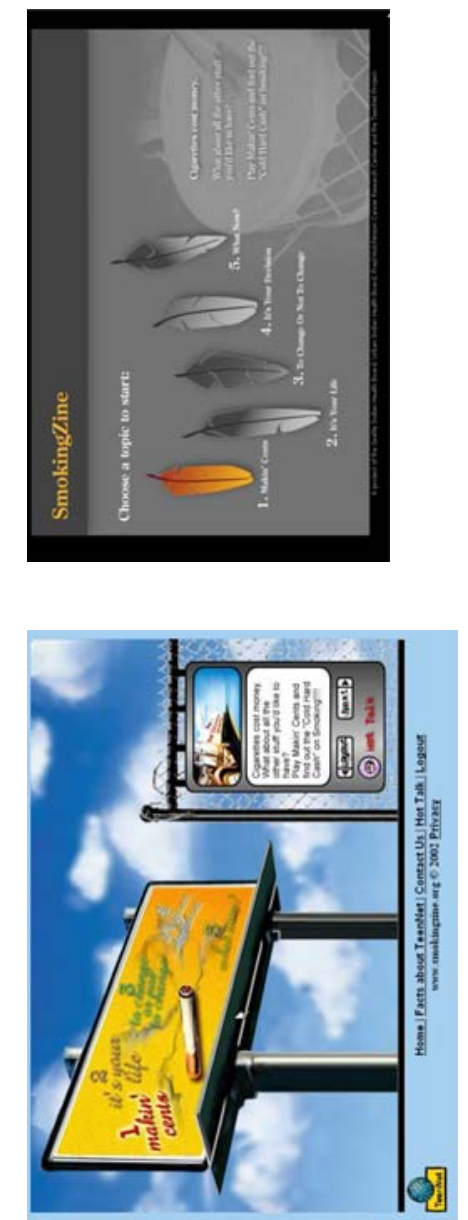

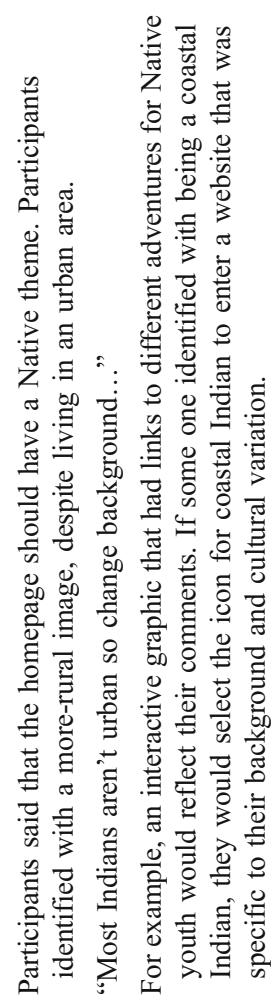

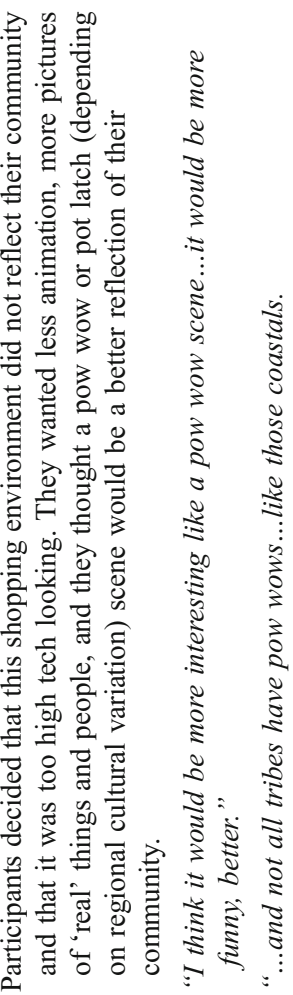
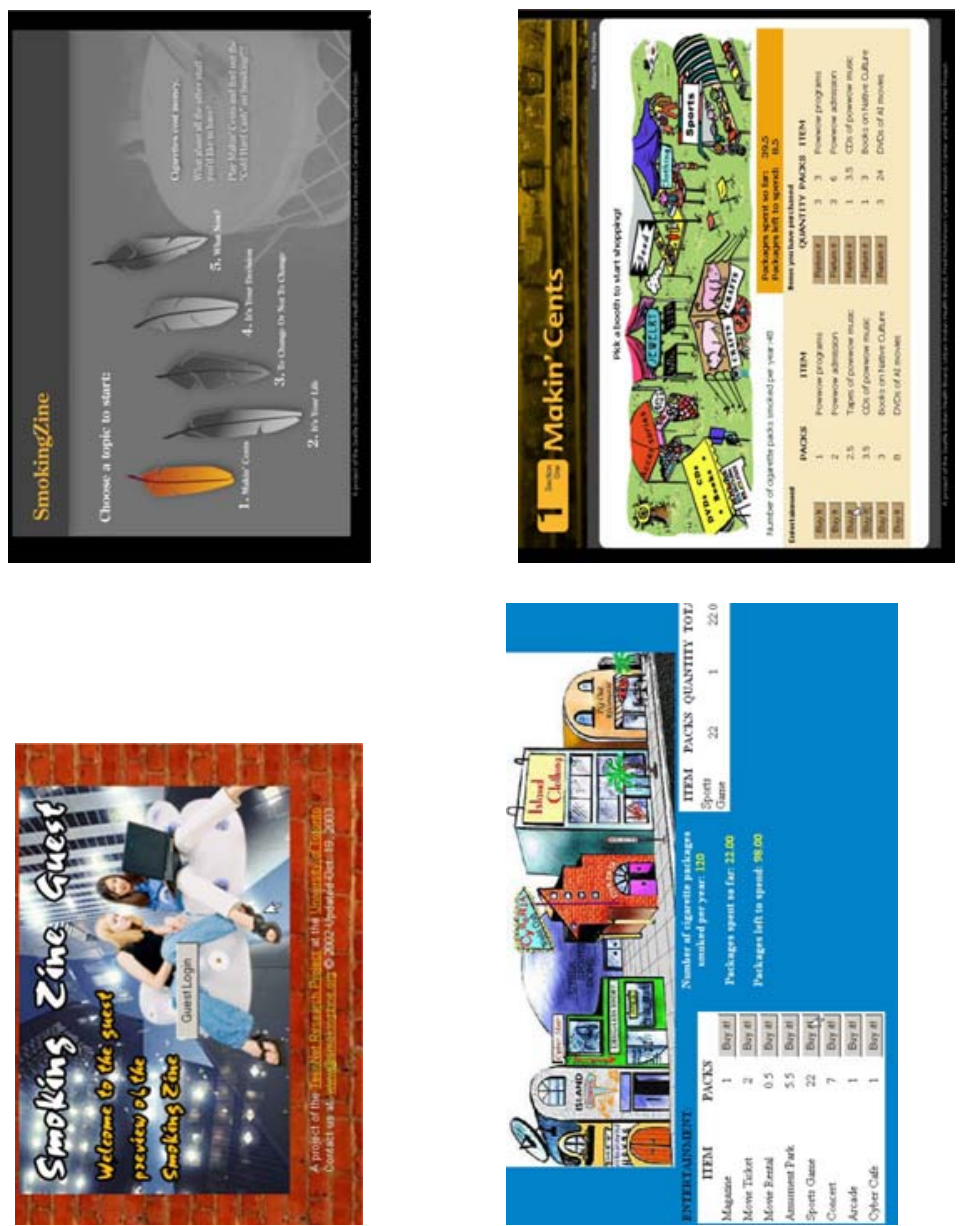

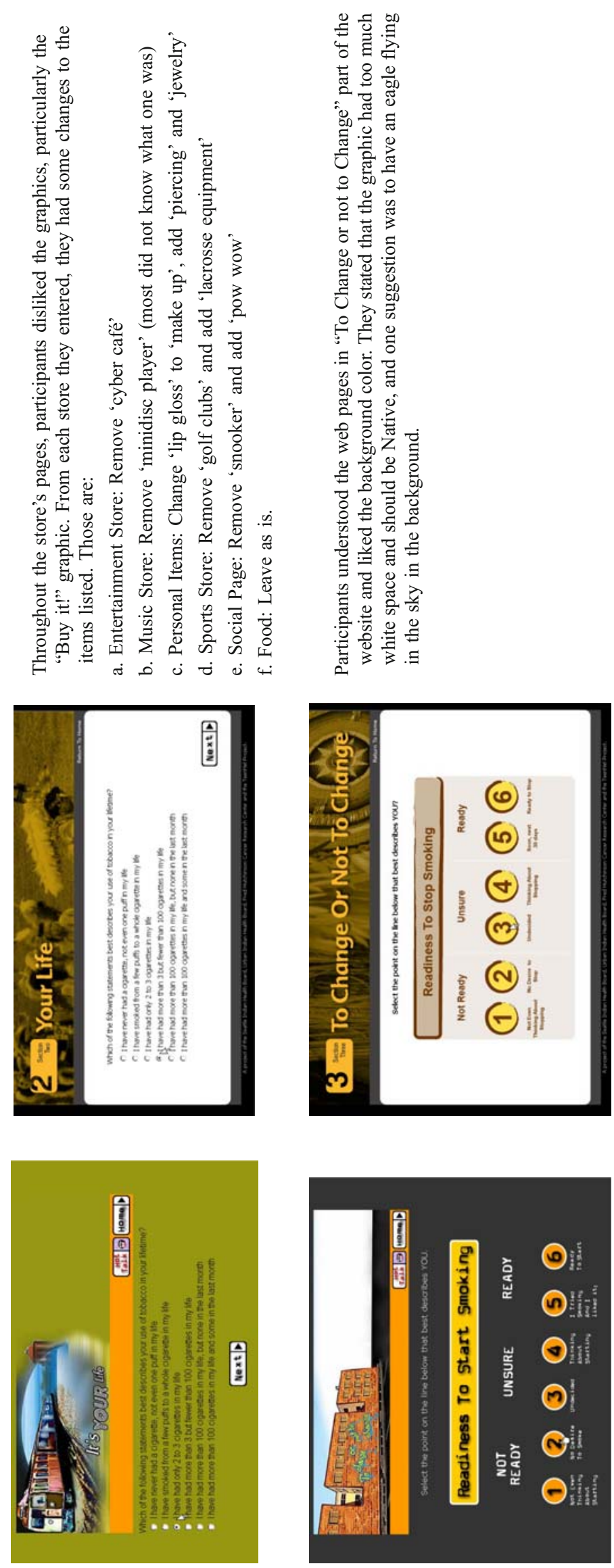
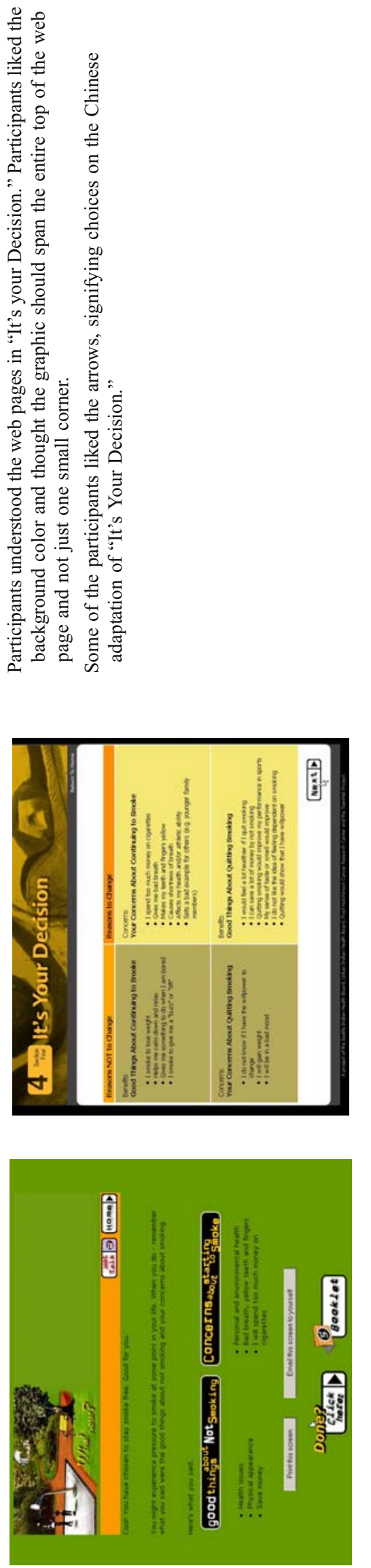
described here have the potential to be cost effective interventions to reduce smoking among Native youth.

Still, the work and results of this study are encouraging, in that we can now move ahead with a full-scale test of this intervention package. This test will involve multiple youth, randomization to intervention or comparison (possibly delayed control) status, and follow-up of youth randomized to these conditions to record relevant data. Therefore, the next step in this research will be to create an opportunity for this type of testing. The future research should include both urban and rural AI/AN youth, and should focus on smoking and tobacco use as acceptable outcomes. Given the relatively high quality of recent smoking prevention and cessation studies in youth, group randomization should be considered if the intervention is to be delivered in schools.

In Washington State, $60 \%$ of high school smokers in are motivated to quit but only $3 \%$ achieve their intention over 12 months [55], suggesting that adolescent smokers recognize the need to quit and that intervening early could avert later smoking. Our data demonstrate the need for increased tobacco control research to identify successful programs for use in young urban AI/ANs.

\section{References}

1. American Indian Health Commission for Washington State \& the Washington State Department of Health (2003) Working together to build a healthy future: the 2003 American Indian health care delivery plan web site. Available at: http://www.aihc-wa.org/Issues/ Documents/AIHCDP/2003_AIHCDP/AIHCDP 2003_Final_PDF. pdf. Accessed on 3 Sept 2008

2. Centers for Disease Control and Prevention (1997) Cigarette smoking among adults - United States. Morb Mort Wkly Rep 48:993-6

3. Centers for Disease Control and Prevention (2000). American Indians and Alaska Natives and Tobacco Web site. Available at: http://www.cdc.gov/tobacco/sgr/sgr_1998/sgr-min-fs-nat.htm. Accessed on 29 Aug 2008

4. Welty TK, Lee ET, Yeh J, Cowan LD, Go O, Fabsitz RR et al (1995) Cardiovascular disease risk factors among American Indians. The strong heart study. Am J Epidemiol 142:269-287

5. Johnson KM, Lando HA, Schmid LS, Solberg LI (1997) Project: outcome of smoking cessation strategies in four urban Native American clinics. Giving American Indians no-smoking strategies. Addict Behav 22:207-218

6. Goldberg HI, Warren CW, Oge LL, Helgerson SD, Pepion DD, LaMere E et al (1991) Prevalence of behavioral risk factors in two American Indian populations in Montana. Am J Prev Med 7:155160

7. King TK, Borrelli B, Black C, Pinto BM, Marcus BH (1997) Minority women and tobacco: implications for smoking cessation interventions. Ann Behav Med 19:301-313

8. Warner KE (1989) Smoking and health: a 25-year perspective. Am J Public Health 79:141-143

9. US Department of Health and Human Services (1998) Tobacco use among US racial/ethnic minority groups-African Americans, American Indians and Alaska Natives, Asian Americans and
Pacific Islanders, and Hispanics: a report of the surgeon general. DHSS, CDC, Atlanta

10. Centers for Disease Control and Prevention (1992) Cigarette smoking among American Indians, Alaskan Natives-behavioral risk factor surveillance system, 1987-1991. Jama 268:3052-3055

11. South Dakota Youth Risk Behavior Survey Report: Native American (1997) Pierre, SD: SD Department of Education \& Cultural Affairs Office of Comprehensive School Health

12. Nelson DE, Moon RW, Holtzman D, Smith P, Siegel PZ (1997) Patterns of health risk behaviors for chronic disease: a comparison between adolescent and adult American Indians living on or near reservations in Montana. J Adolesc Health 21:25-32

13. Everett S, Sussman M, Ranslow S, Shaughnessy L (2000) Youth risk behavior survey of middle school students attending bureau funded schools, 2000. Bureau of Indian Affairs, Office of Indian Education Programs in Conjunction with the Centers for Disease Control

14. Indian Health Service (2000) Indian Health Service Health Care Improvement Act Web site. Available at: http://info.ihs.gov/ TreatiesLaws/Treaties3.pdf. Accessed on 2 Sept 2008

15. Guilmet GM (1999) Chief Leschi safe futures project final report. Safe and Drug-Free Schools Program, US Department of Education, Washington DC, p 166

16. US Census Bureau (2003) American Indian and Alaska Native Populations Web site. Available at: http://www.census.gov/ population/www/socdemo/race/indian.html. Accessed on 29 Aug 2008

17. Forquera R (2003) Seattle Indian Health Board for The Henry J. Kaiser Family Foundation (2001). Urban Indian Health Web site. Available at: http://www.kff.org/content/2001/6006/6006Revised. pdf. Accessed 3 September 2003

18. Frisby G, Bessell TL, Borland R, Anderson JN (2002) Smoking cessation and the Internet: a qualitative method examining online consumer behavior. J Med Internet Res 4:E8

19. Denny JT, Ginsberg S, Papp D, Browne G, Morgan S, Kushins L et al (2002) Hospital initiatives in promoting smoking cessation: a survey of Internet and hospital-based programs targeted at consumers. Chest 122:692-698

20. Riemsma RP, Pattenden J, Bridle C, Sowden AJ, Mather L, Watt IS et al (2003) Systematic review of the effectiveness of stage based interventions to promote smoking cessation. Bmj 326:1175-1177

21. Ademiluyi G, Rees CE, Sheard CE (2003) Evaluating the reliability and validity of three tools to assess the quality of health information on the Internet. Patient Educ Couns 50:151-155

22. Woodruff SI, Edwards CC, Conway TL, Elliott SP (2001) Pilot test of an Internet virtual world chat room for rural teen smokers. J Adolesc Health 29:239-243

23. Etter JF (2002) Using new information technology to treat tobacco dependence. Respiration 69:111-114

24. Lenert L, Munoz RF, Stoddard J, Delucchi K, Bansod A, Skoczen $S$ et al (2003) Design and pilot evaluation of an internet smoking cessation program. J Am Med Inform Assoc 10:16-20

25. Feil EG, Noell J, Lichtenstein E, Boles SM, McKay HG (2003) Evaluation of an Internet-based smoking cessation program: lessons learned from a pilot study. Nicotine Tob Res 5:189-194

26. Etter JF, le Houezec J, Landfeldt B (2003) Impact of messages on concomitant use of nicotine replacement therapy and cigarettes: a randomized trial on the Internet. Addiction 98:941-950

27. Skinner H, Morrison M, Bercovitz K, Haans D, Jennings MJ, Magdenko L et al (1997) Using the Internet to engage youth in health promotion. Promot Educ 4:23-25

28. Ribisl KM, Kim AE, Williams RS (2001) Web sites selling cigarettes: how many are there in the USA and what are their sales practices? Tob Control 10:352-9 
29. Boston University (2008) Quitnet Web site. Available at: http://www. quitnet.com/p/bu/Boston_University_Intro/Boston_University_ Intro.htm. Accessed on 31 October 2008

30. Washington State Department of Health (2008). Quitline.com Web site. Available at: http://www.quitline.com/. Accessed 2008

31. Mississippi State Department of Health (2008). Mississippi Tobacco Quitline Web site. Available at: http://www.quitlinems. com/. Accessed 2008

32. National Center For Chronic Disease Prevention and Health Promotion (2003) TIPS- Tobacco information and prevention source web site. Available at: http://www.cdc.gov/tobacco/how2quit.htm. Accessed 29 Aug 2008

33. Schwartz W. ERIC Clearinghouse on Urban Education (2000) Kidsource online: parent guide: a guide to youth smoking prevention policies and programs web site. Available at: http:// www.kidsource.com/kidsource/content4/smoking.prevention.html. Accessed 29 Aug 2003

34. University of California, San Diego (2003) NoButts: California smokers helpline web site. Available at: http://nobutts.ucsd.edu/. Accessed on 29 Aug 2008

35. National Health Service, UK (2008). Smokefree web site. Available at: http://gosmokefree.nhs.uk/what-suits-me/nhs-smoking-helpline/. Accessed 2008

36. University of Toronto (2003) Smoking Zine web site. Available at: http://www.teennetproject.org/project_websites_smoking.html. Accessed on 11 Sept 2008

37. Skinner H, Maley O, Smith L, Morrison M, Chirrey S (2001) New Frontiers: using the internet to engage teens in substance abuse prevention and treatment. In: Monte P, Colby S (eds) Adolescence, alcohol, and substance abuse: reaching teens through brief interventions. Guilford Press, New York, pp 297-318

38. Skinner H, Biscope S, Poland B (2003) Quality of internet access: barrier behind internet use statistics. Soc Sci Med 57:875-880

39. Skinner HA, Maley O, Norman CD (2006) Developing internetbased eHealth promotion programs: the Spiral Technology Action Research (STAR) model. Health Promot Pract 7:406-417

40. University of Toronto (2003) TeenNet Project Web site. Available at: http://www.teennetproject.org/index.html. Accessed on $11 \mathrm{Sept}$ 2008

41. Schinke SP, Singer B, Cole K, Contento IR (1996) Reducing cancer risk among Native American adolescents. Prev Med 25:146-155

42. Botvin JD (2002) Allina Web site offers visitors a chance for conversation. One of the nation's 'most wired', it also is quite accessible. Profiles Healthc Mark 18:27-32
43. Botvin GJ, Schinke SP, Orlandi MA (1995) School-based health promotion: substance abuse and sexual behavior. Appl Prev Psychol 4:167-184

44. Botvin GJ, Schinke SP, Epstein JA, Díaz T, Botvin EM (1995) Effectiveness of culturally focused and generic skills training approaches to alcohol and drug abuse prevention among minority adolescents: two-year followup results. Psychol Addict Behav 9:183-194

45. Tobler N, Stratton H (1997) Effectiveness of school based drug prevention programs: a meta-analysis of the research. J Prim Prev $18: 71-128$

46. Tobler N, Roona M, Ochshorn P, Marshall DG, Streke AV, KM S (2000) School-based adolescent drug prevention programs: 1998 meta-analysis. J Prim Prev 20:275-336

47. Unger JB, Ritt-Olson A, Teran L, Huang T, Hoffman BR, Palmer P (2002) Cultural values and substance use in a multiethnic sample of California adolescents. Addiction Research \& Theory 10:257-279

48. Unger JB, Chou CP, Palmer PH, Ritt-Olson A, Gallaher P, Cen S et al (2004) Project FLAVOR: 1-year outcomes of a multicultural, school-based smoking prevention curriculum for adolescents. Am J Public Health 94:263-265

49. Philips S (1972) Participant structures and communicative competence: Warm Spring children in community and classroom. In: Cazden C, John V, Hymes D (eds) Functions of language in the classroom. Waveland, Prospect Heights, IL, pp 370-394

50. Reyhner J (2002) American Indian \& Alaska Native Education: an overview. American Indian Education Web site. Available at: http://jan.ucc.nau.edu/ jar/AIE/Ind_Ed.html. Accessed on 31 Oct 2008

51. Rougas ME (2000) A cognitive profile of Mohawk adolescents using the Woodcock-Johnson Tests of Cognitive AbilityRevised. Unpublished Dissertation. State University of New York, Albany, NY

52. Riding R, Rayner S (1998) Cognitive styles and learning strategies: Understanding style differences in learning and behaviour. David Fulton Publishing, London

53. Morton LL, Allen JD, Williams NH (1994) Hemisphericity and information processing in North American Native (Ojibwa) and non-native adolescents. Int J Neurosci 75:189-202

54. Indian Nations at Risk Task Force (1991) Final report. U.S. Department of Education, Washington, D.C

55. Burt RD, Peterson AV Jr (1998) Smoking cessation among high school seniors. Prev Med 27:319-327 